Check for updates

Cite this: RSC Adv., 2018, 8, 37795

Received 30th July 2018

Accepted 1st October 2018

DOI: $10.1039 / c 8 r a 06419 a$

rsc.li/rsc-advances

\section{A microRNA-4516 inhibitor sensitizes chemo- resistant gastric cancer cells to chemotherapy by upregulating ING4}

\author{
Jun-bao Liu, (D) $\dagger^{a}$ Dan Chen, $\uparrow^{\mathrm{b}}$ Hai-xia Liu, $\uparrow^{\mathrm{c}}$ Huan-huan Sha, $\dagger^{\mathrm{bd}}$ Dan Song, $\dagger^{\mathrm{e}}$ \\ Ting-ting Bao, $\dagger^{f}$ Jian-wei $\mathrm{Lu}^{\star g}$ and Chen $\mathrm{Yu}^{\star f}$
}

Objective MicroRNA (miRNA) is an important factor in the regulation of gene transcription. This study was aimed at investigating the role of miR-4516 in the chemoresistance of gastric cancer cells. Methods miR4516 expression levels were measured in gastric cancer cell line SGC7901 and in 5-fluorouracil (5-FU)resistant SGC7901 cells (SGC7901/5-FU) via microarray analysis and RT-PCR. A miR-4516 inhibitor and negative controls were transfected into SGC7901/5-FU cells. A miR-4516 mimic and negative controls were transfected into SGC7901 cells. CCK8 and flow-cytometric assays were performed to evaluate the sensitivity of SGC7901/5-FU cells to 5-FU. Western blot experiments detected the expression of Bcl-2, Bax, Caspase-3, P-gp and ING4 protein. Results Additionally, ING4 was demonstrated to be downregulated in SGC7901/5-FU cells and inversely correlated with miR-4516 expression. Rescue experiments revealed that overexpression of ING4 attenuated the inhibitory effects of miR-4516 on the proliferation of gastric cancer cells. ING4 was predicted to be a potential target of miR-4516. Synergism of the inhibitory effects correlated with a reduction in the expression of the anti-apoptotic protein Bcl-2 and the drug resistance-related protein $\mathrm{P}$-gp as well as strong expression of apoptosis-related proteins (Bax, Caspase-3). Thus, a miR-4516 inhibitor sensitized gastric cancer SGC7901/5-FU cells to 5-FU by enhancing apoptosis. We then corroborated these results with in vivo experiments. Conclusion We found that miR-4516 might be a potential therapeutic target in chemo-resistant gastric cancer.

\section{Introduction}

Gastric cancer is one of the major causes of death in the world..$^{1,2}$ Its incidence correlates with several risk factors, such as age, race, gender, and location. For example, gastric cancer is

\footnotetext{
${ }^{a}$ Traditional Chinese Medicine Department, People's Hospital of Henan Province, People's Hospital of Zhengzhou University, Zhengzhou, Henan, 450003, China. E-mail:41186709@qq.com; lujw@medmail.com.cn

${ }^{b}$ Research Center of Clinical Oncology, Jiangsu Cancer Hospital \& Jiangsu Institute of Cancer Research \& The Affiliated Cancer Hospital of Nanjing Medical University, Nanjing, Jiang Su, 210000, China

${ }^{\circ}$ Emergency Department, The Second Affiliated Hospital of Nanjing University of Chinese Medicine, Nanjing, Jiang Su, 210017, China

${ }^{d}$ The Forth Clinical School of Nanjing Medical University, Nanjing, Jiang Su, 21000o, China ${ }^{e}$ Department of Radiotherapy, Jiangsu Cancer Hospital \& Jiangsu Institute of Cancer Research \& The Affiliated Cancer Hospital of Nanjing Medical University, Nanjing, Jiang Su, 210000, China

${ }^{{ }_{\text {Department }}}$ of Integrated TCM \& Western Medicine, Jiangsu Cancer Hospital \& Jiangsu Institute of Cancer Research \& The Affiliated Cancer Hospital of Nanjing Medical University, Nanjing, Jiang Su, 210000, China. E-mail: 41186709@qq.com

${ }^{g}$ Department of Medicine, Jiangsu Cancer Hospital \& Jiangsu Institute of Cancer Research \& The Affiliated Cancer Hospital of Nanjing Medical University, Nanjing, Jiang Su, 210000, China. E-mail: lujw@medmail.com.cn

$\dagger$ Jun-bao Liu, Dan Chen, Hai-xia Liu, Huan-huan Sha, Dan Song and Ting-ting Bao contributed equally to this work.
}

more common in such countries as Korea and Japan. Surgical resection remains the primary treatment of this cancer; ${ }^{3}$ on the other hand, the resistance of gastric cancer cells often leads to chemotherapy failure, which is a key problem affecting the longterm survival of patients with gastric cancer. An effective way to improve the efficacy of gastric cancer treatment in patients is to explore the mechanism of gastric cancer resistance and to find molecular markers for reversing the drug resistance of gastric cancer. These patients, in particular, require aggressive treatment involving radiation and/or chemotherapy. For example, antimetabolic drugs such as 5-fluorouracil (5-FU) have been widely used to treat gastric cancer. Numerous attempts have been made to improve the response of these patients to chemotherapy. Specifically, resistance to chemotherapeutic drugs can hinder the prolongation of survival time in patients with gastric cancer. ${ }^{4}$ Thus, finding a way to re-sensitize chemoresistant gastric cancer cells and to overcome this drug resistance should have a significant clinical impact.

MicroRNA (miRNA) is an endogenous noncoding small RNA molecule that regulates the translation or degradation of target mRNAs. ${ }^{5,6}$ This protein noncoding RNA family is widespread in the biological world. It has been found that miRNA regulates expression of one-third mRNA. Because the relation between miRNA and cancer is gradually revealed, there is evidence that 
the sensitivity of cells to antitumor drugs is affected by miRNA. ${ }^{7}$ Some experiments have shown that certain miRNAs, including miR-25, miR-20a, and miR-623 are associated with a poor prognosis of cancer and have been reported to be significantly upregulated in recurrent gastric carcinoma. ${ }^{8-12}$ Therefore, we hypothesized that gastric cancer resistance may be related to abnormal expression of miRNAs. In addition, a lot of evidence has revealed that miRNA is involved in gastric cancer initiation and progression, including MDR. ${ }^{13-15}$ We thus speculated that dysregulation of the miRNA that regulates ING4 expression might be the main cause of drug resistance.

In the present study, we demonstrated that miR-4516, which is overexpressed in multidrug-resistant gastric cancer cell line SGC7901/5-FU, suppresses ING4 expression by targeting the $3^{\prime}$ untranslated region (UTR) of its mRNA. Additionally, we found that a miR-4516 inhibitor suppressed gastric cancer MDR, whereas upregulation of miR-4516 had the opposite effects. We showed that ING4 was overexpressed in chemo-sensitive gastric cancer cells compared with chemo-resistant ones. Decreased expression of ING4 predicted poor survival. Besides, we found that upregulation of ING4 enhanced chemosensitivity to 5-FU in SGC7901/5-FU cells. Moreover, we propose that the miR-4516ING4 axis regulates gastric cancer MDR through apoptosisrelated molecules (Bcl-2, Bax, caspase 3).

\section{Materials and methods}

\section{Cell culture and drugs}

Human gastric cancer cell line SGC7901 was kindly provided by the First Affiliated Hospital of Nanjing Medical University (Nanjing, China). Human multidrug-resistant gastric cancer cell line SGC7901/5-FU was purchased from Sai qi Biological Engineering Co., Ltd. (Shanghai, China). The cells were propagated in the RPMI 1640 medium (Gibco BRL, Carlsbad, CA, USA) supplemented with $10 \%$ of fetal of bovine serum, streptomycin $\left(100 \mu \mathrm{g} \mathrm{mL}^{-1}\right)$, and penicillin $\left(100 \mathrm{U} \mathrm{mL}^{-1}\right)$ in a humidified atmosphere containing $5 \%$ of $\mathrm{CO}_{2}$. For the SGC7901/5-FU cell line, the medium was supplemented with $1 \mathrm{mg} \mathrm{mL}^{-1} 5$-FU (5FU; Sigma-Aldrich, St. Louis, MO, USA). The dilutions for all the reagents were freshly prepared before each experiment.

\section{Drug sensitivity assa in vitro}

Drug sensitivity was assessed. Briefly, $5 \times 10^{3}$ cells were seeded in 96-well plates, the cells were mixed and cultured in an incubator for 4 to 6 hours. After the cells were attached, the medium was replaced by the serum-containing medium. The various concentrations of 5-FU or miR-4516 inhibitor was added to each well. After 24 hours of incubation, the CCK-8 (Beyotime Institute of Biotechnology, Haimen, China) assay was performed. Inhibition rates and IC50 values were calculated. All experiments were conducted in triplicate.

\section{Flow cytometric analysis of cell apoptosis}

A total of $5 \times 10^{5}$ cells were seeded in 6-well plates and cultured at $37{ }^{\circ} \mathrm{C}$ and $5 \% \mathrm{CO}_{2}$. After 48 hours of transfection, the cells were further cultured with a miR-4516 inhibitor $\left(100 \mathrm{nmol} \mathrm{L}^{-1}\right)$ for $24 \mathrm{~h}$. The cells were harvested, washed with ice-cold phosphate-buffered saline (PBS), and collected into a $1.5 \mathrm{~mL}$ tube. Apoptosis was then detected using the Annexin V-FITC Apoptosis Detection Kit (BD Biosciences). In brief, $300 \mu \mathrm{L}$ of $1 \times$ binding buffer was added to a $1.5 \mathrm{~mL}$ tube, gently mixed with the cells, and $5 \mu \mathrm{L}$ of annexin $\mathrm{V}$ and $5 \mu \mathrm{L}$ of propidium iodide (PI) were added. The cells were incubated at room temperature in the dark for $15 \mathrm{~min}$, and apoptosis was analyzed on a flow cytometer (Beckman Coulter, Inc., Brea, CA, USA). Each assay was performed in triplicate.

\section{Cell transfection and treatment}

A suitable volume of serum-free medium was added into a transfection tube. Next, a plasmid expressing miR-4516, a miR-4516 inhibitor, or their corresponding control was transfected into gastric cancer cells using Lipofectamine 2000 (Invitrogen). In some experiments, the miR-4516 inhibitor was co-transfected with a plasmid expressing ING4-specific small interfering RNA (siING4), and then the tube was shaken after addition of the appropriate volume of the transfection reagent. The cultivation of the cells was continued. The overexpression and knockdown efficiency rates were determined by RT-PCR and/or western blot analysis.

\section{A luciferase reporter assay}

The 3 '-UTR of human ING4 cDNA containing the putative target site for mature miR-4516 was inserted into the multiple cloning site of the pmirGLO Luciferase vector (Promega). A mutant ING4 3 '-UTR lacking the binding site for miR-4516 was likewise cloned into the pmirGLO Luciferase vector (Promega). The wildtype (Wt) or mutant construct was transfected into SGC7901/5FU cells $\left(6 \times 10^{4}\right.$ cells per well), which were incubated in 24 -well plates, and either a miR-4516 mimic or control mimic (GenePharma, Suzhou, China) was transfected into the cells by means of Lipofectamine 2000 (Invitrogen). At $48 \mathrm{~h}$ after transfection, the cells were assayed for relative luciferase activity using the Dual-Luciferase Reporter Assay System (Promega) to compare with a no-load control.

\section{Quantitative RT-PCR}

A TRIzol Kit (Bioline) was employed to prepare total RNA from SGC7901 or SGC7901/5-FU cells. The cycling conditions of PCR amplification were as follows: $94{ }^{\circ} \mathrm{C}$ for $10 \mathrm{~min}$; 40 cycles of $94{ }^{\circ} \mathrm{C}$ for $15 \mathrm{~s}, 60{ }^{\circ} \mathrm{C}$ for $1 \mathrm{~min}, 72{ }^{\circ} \mathrm{C}$ for $1 \mathrm{~min}$; then $72{ }^{\circ} \mathrm{C}$ for 5 min. RT-PCR was performed in triplicate on an ABI Step One Plus Real-Time PCR system (Applied Biosystems) with $2 \mathrm{mg}$ of each cDNA sample and the SYBR® Select Master Mix (Applied Biosystems). Expression levels were normalized to an internal standard. The PCR primers are presented in Fig. 1A. Relative gene expression was analyzed by the $2^{-\Delta \Delta C_{t}}$ method, and the results are expressed as a change relative to control values.

\section{Western blot assay}

Cells were lysed with the mammalian protein extraction reagent RIPA (Beyotime, Beijing, China). Proteins in $\sim 50 \mu \mathrm{g}$ of a protein 
A

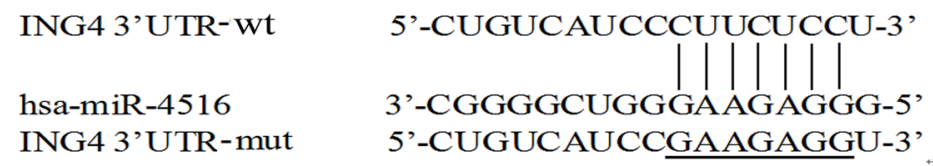

B

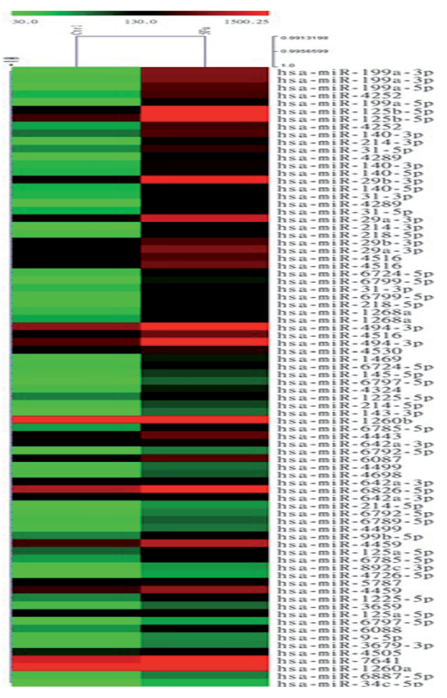

$\mathrm{C}$

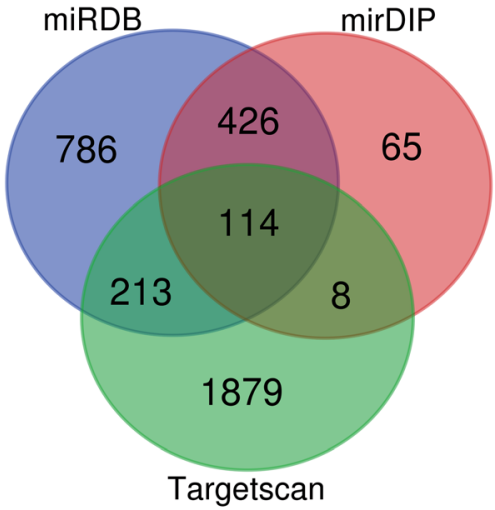

$\mathrm{D}$
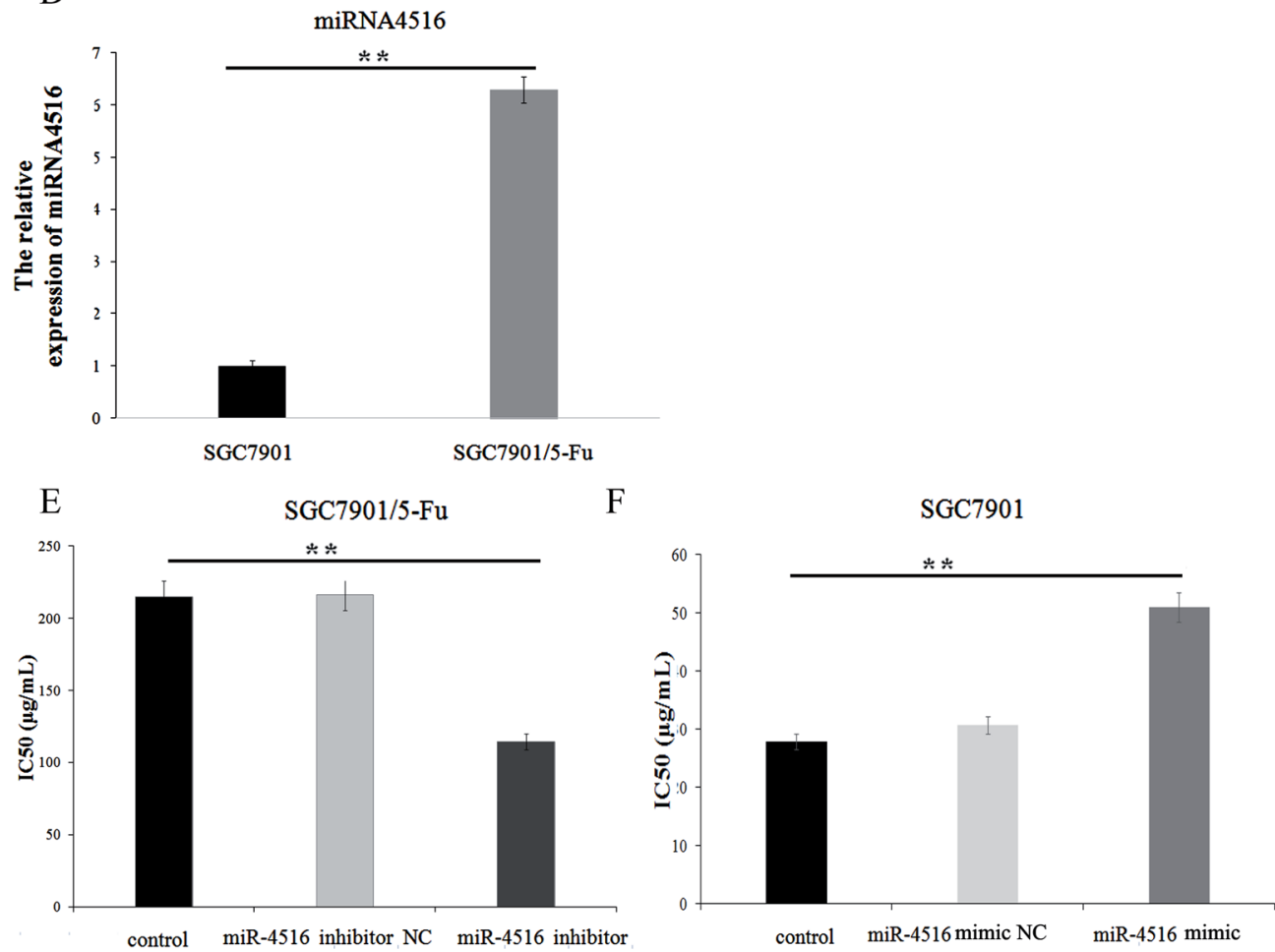

Fig. 1 miR-4516 is downregulated in GC tissues and cell lines (A) bioinformatic analysis based on TargetScan software revealed that the $3^{\prime}$ - UTR of ING4 mRNA harbored a putative site complementary to the seed sequence of miR-4516. (B) Hierarchical clustering analysis of the differentially expressed miRNAs in SGC7901/5-FU compared with SGC7901/5-FU without intervention. Each row represents the expression of miRNA and each column represents a sample: red color for upregulation, green for downregulation. (C) The consensus target genes of each differentially expressed miRNA. The target genes of the miR-4516 were predicted with three online tools (miRDB, Targetscan and mirDIP). (D) RT-qPCR were applied to measure the mRNA expression levels of miRNA-4516 in SGC7901/5-FU cells and SGC7901 cells, respectively. *P<0.05 compared with normal tissues. (E) The proliferation of SGC7901/5-FU cells were determined through CCK-8 assay following transfection with miR-4516 inhibitor or miR-NC. **P< 0.01 compared with miR-NC. (F) The proliferation of SGC-7901 cells was determined through CCK- 8 assay following transfection with miR-4516 mimic or miR-NC. $* * P<0.01$ compared with miR-NC. 
A
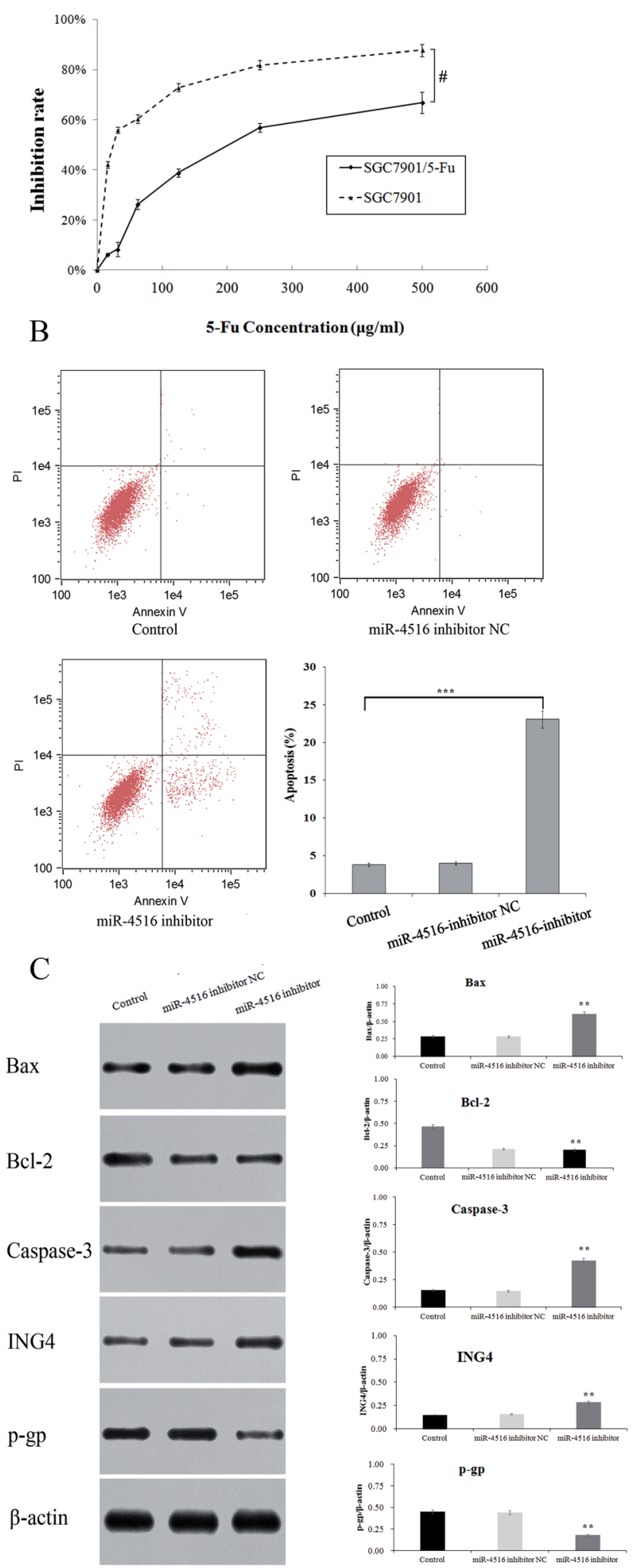

Fig. 2 miR-4516 inhibits the proliferation and enhances the chemosensitivity of SGC-7901/5-FU cells to 5-FU (A) cell inhibition ratios at increasing concentrations of 5-FU in SGC7901/5-FU cells or SGC7901 cells. The cell inhibition ratio was determined by the CCK- 8 assay. (B) SGC7901/5-FU cells were transfected with miR-4516 inhibitor or miR$\mathrm{NC}$ and incubated $48 \mathrm{~h}$ after transfection. Cell apoptosis was analyzed through flow cytometry to examine the cell apoptosis rate. $* P<0.05$ extract were separated by SDS-PAGE in a $10 \%$ gel, transferred to a $0.22 \mu \mathrm{m}$ nitrocellulose membrane (Sigma), and incubated with specific antibodies. Different groups of proteins were subjected to electrophoresis, transferred, and exposed. Autoradiograms were quantified by densitometry in the Quantity One software (Bio-Rad, Carlsbad, CA, USA). An anti- $\beta$-actin antibody served as a control, and rabbit anti-ING4, anti-Bcl-2, anti-Bax, anti-caspase 3, and anti-P-gp antibodies were provided by Cell Signaling Technology (Danvers, MA, USA).

\section{Animal experiments}

SGC7901/5-FU cells stably expressing the miR-4516 inhibitor combined with 5 -FU $\left(20 \mathrm{mg} \mathrm{kg}^{-1}\right)$, or 5 -FU $\left(20 \mathrm{mg} \mathrm{kg}^{-1}\right)$ alone were injected subcutaneously into 5 week-old male nude mice $(n$ $=6 ; 3 \times 10^{6}$ cells per mouse). After $\sim 2$ weeks, the subcutaneous xenograft of nude mice became noticeable. The mice were housed in a specific pathogen-free (SPF) room with laminar flow. Tumor size was measured every 3 days. At 15 days after cell injection, the mice were euthanized, and xenograft tumors were excised and weighed. The animal experiments were performed according to the institutional guidelines.

\section{Statistical analysis}

All measurement data are expressed as the mean $\pm \mathrm{SD}$. The significance of differences in clinical data regarding ING4 expression was determined by Student's $t$ test. All experimental data were analyzed in the SPSS 16.0 statistical software (SPSS Inc., Chicago, IL, USA) for Windows. The significance level was set to $P<0.05$. Each experiment was repeated three times.

\section{Ethical statement}

This study was performed in strict accordance with the Good Clinical Practice (GCP promulgated on 2017.01.18) by State Administration of Food and Drug Administration, and was approved by the centralab and ethics committee of Jiangsu Cancer Hospital.

\section{Results}

\section{MiR-4516 activation is involved in chemoresistance of gastric cancer cells}

Dysfunction of miRNAs can be detected in 5-FU-resistant cells. Many experiments have revealed that miRNA is associated with acquired drug resistance in gastric cancer cells. Therefore, we used miRNA microarray detection to analyze the expression level of miRNA in drug-resistant gastric cancer cells and sensitive cell lines. We compared the normal proportions and difference multiplier of miRNA microarray hybridization signals in two cell lines and constructed a heatmap by

compared with miR-NC. (C) Western blotting of P-gp, Bax, Bcl-2, Caspase-3 and ING4 in SGC7901/5-FU cells after transfection with miR-4516 inhibitor. $\beta$-Actin was used as an internal control. Schematic representation of the miR-4516/ING4 axis as multidrug resistance (MDR) regulator in GC. $* P<0.05$ compared with miR-NC. 
A

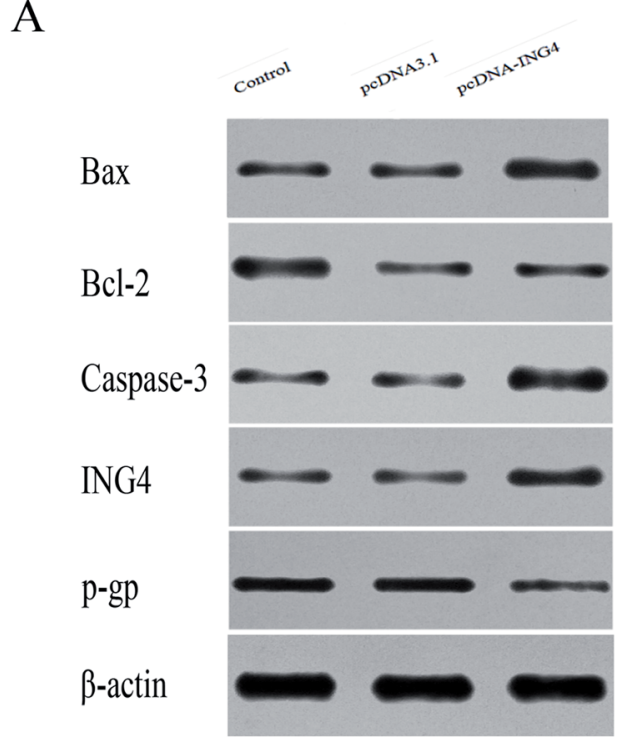

$\mathrm{B}$

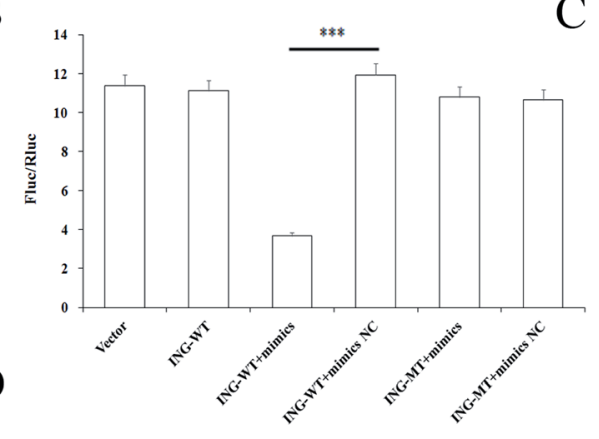

$\bar{\alpha}$
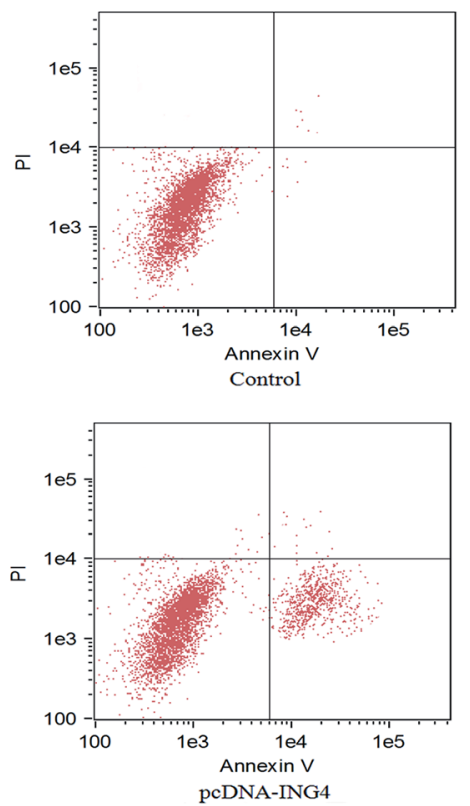
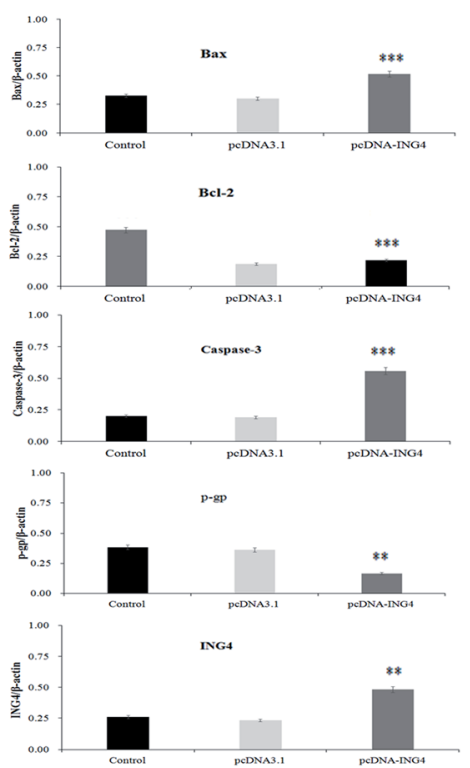

$\mathrm{C}$
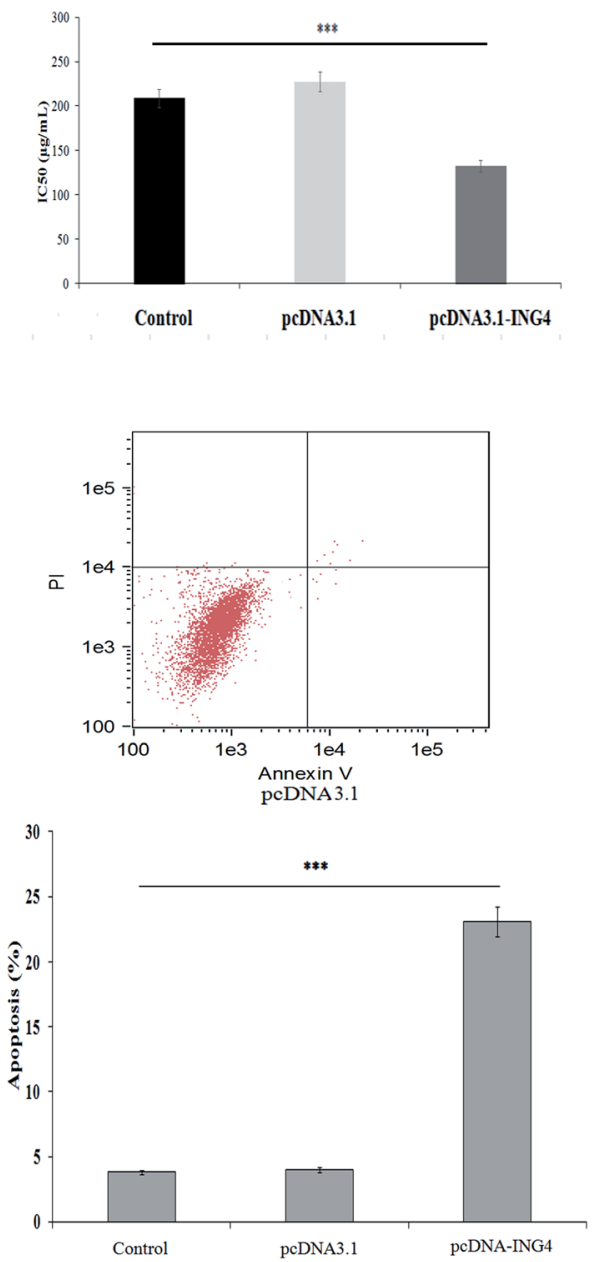

Fig. 3 miR-4516 directly targets ING4 mRNA 3'-UTR in gastric cancer (A) western blot was performed to determine the protein levels of P-gp, Bax, Bcl-2 Caspase-3 and ING4 in SGC-7901/5-FU cells transfected with pcDNA3.1-ING4 or pcDNA3.1. *P<0.05 compared with ING4 plasmid.

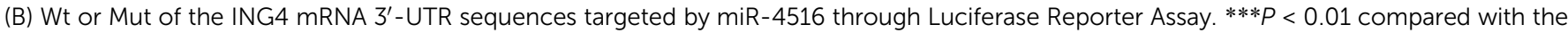
miR-Ctrl group. (C) The proliferation of SGC-7901/5-FU cells were determined through CCK-8 assay following transfection with pCDNA3.1ING4 or pcDNA3.1. ***P<0.001 compared with pCDNA3.1. (D) SGC7901/5-FU cells were transfected with pc-DNA-ING4 or pc-DNA3.1 and incubated $48 \mathrm{~h}$ after transfection. Cell apoptosis was analyzed through flow cytometry to examine the cell apoptosis rate. *** $P<0.001$ compared with pc-DNA3.1. 
A

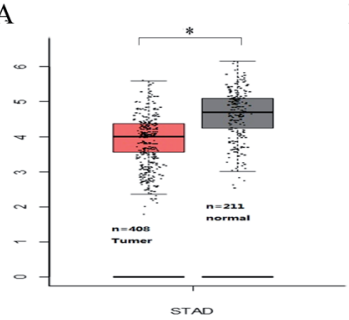

$\mathrm{C}$

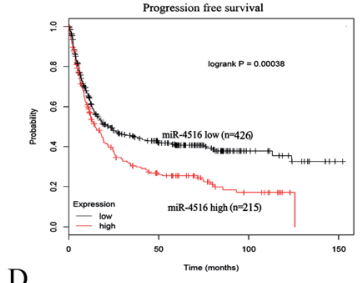

$\mathrm{D}$

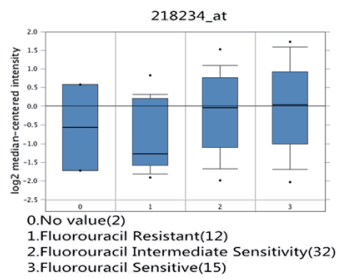

$\mathrm{F}$
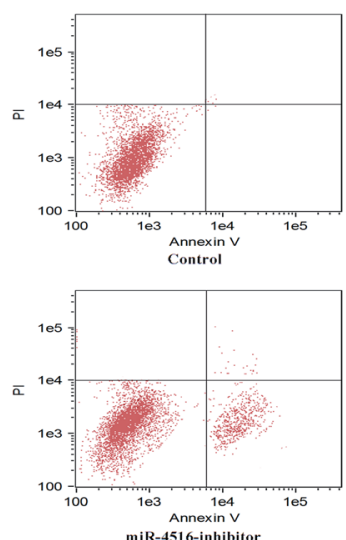

in-4516-inhibitor
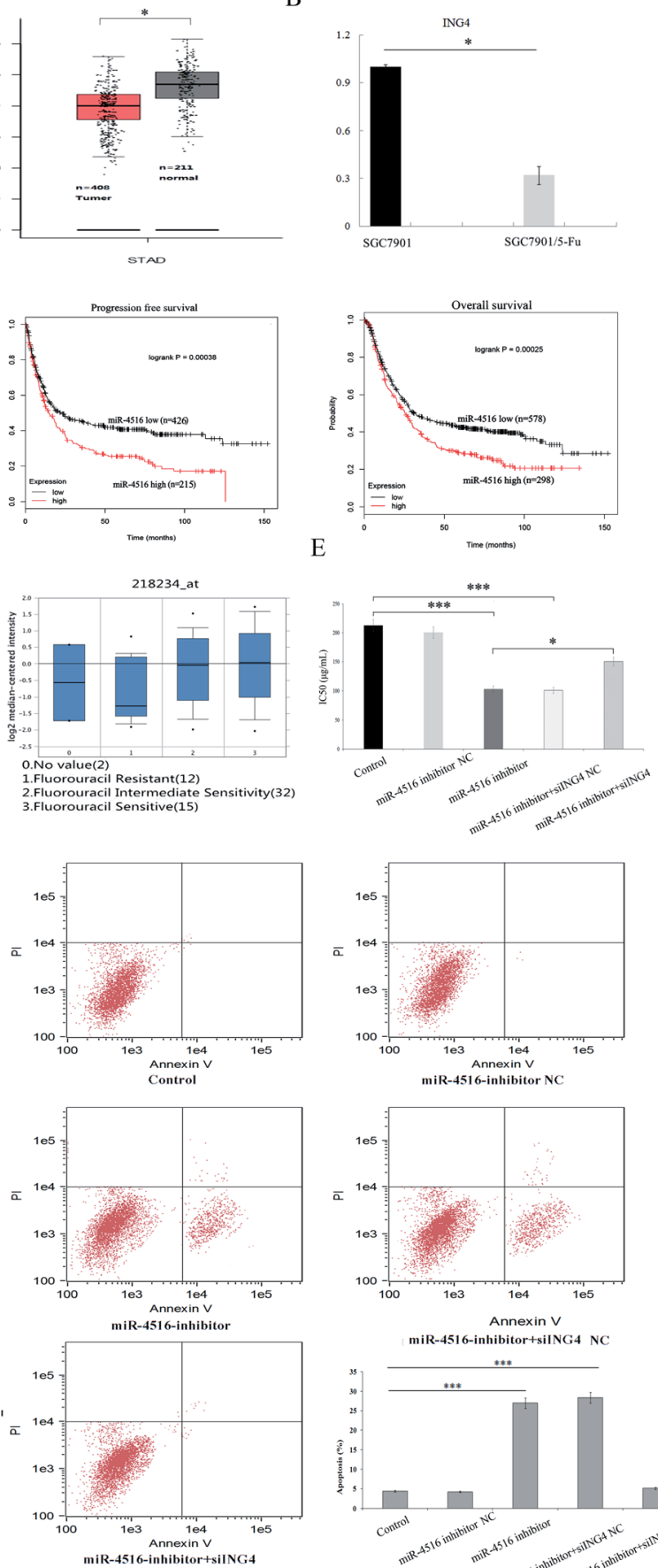

miR-4516-inhibitor NC
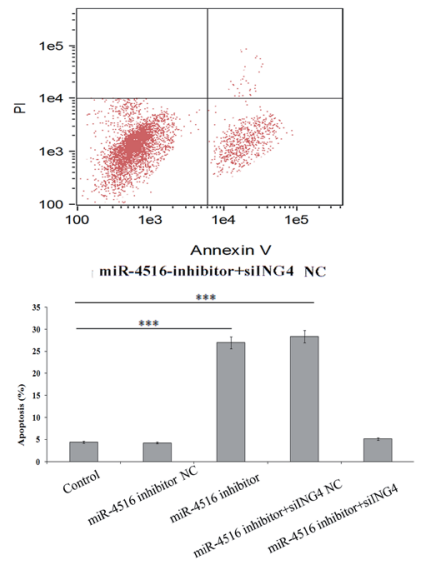

Fig. 4 ING4 overexpression in GC tissues is inversely correlated with miR-4516 level. (A) RT-qPCR were applied to measure the mRNA expression levels of ING4 in GC tissues and adjacent normal gastric tissues ( $n=408$ ), respectively. $* P<0.05$ compared with normal tissues. (B) Expression level of ING4 mRNA in chemo-sensitive and chemo-resistant GC tissues was measured using quantitative RT-PCR from oncomine database in compendia cell line. GAPDH was used as an internal control and the fold change was calculated by $2^{-\Delta \Delta C_{t}} . * P<$ 0.05. (C) Association of ING4 with overall survival and Progression free survival in GC patients from Kaplan-Meier plotter. (D) Level of ING4 mRNA was significantly decreased from 5-fluorouracil sensitive to 5fluorouracil resistance in the probes (218234_at) in dataset. (E) The cell inhibition ratio was determined by the CCK- 8 assay. Cell inhibition ratios at IC50 values for 5-FU were observed after ING4 expression

clustering miRNA. As depicted in Fig. 1B, some miRNAs were differentially expressed between cell lines SGC7901 and SGC7901/5-FU, and we found that 47 miRNAs were upregulated, while 55 miRNAs were downregulated. As shown in Fig. 1D, miR-4516 expression was 6.29-fold higher in SGC7901/5-FU cells than SGC7901 cells. According to our previous results, miR-4516 is most differentially expressed in drug-resistant cells. Accordingly, for this follow-up study, we selected miR-4516 as a target, and we confirmed that miR-4516 was downregulated by $52.84 \%$ in drug-resistant cells after treatment with miR-4516 inhibitor (Fig. 1E).

\section{MiR-4516 inhibitor decreased cell proliferation and enhanced sensitivity to 5 -FU}

We performed a CCK-8 assay to determine the effect of 5-FU on the viability 5-FU-resistant and -sensitive cells. The two gastric cancer cell lines (SGC7901/5-FU and SGC7901) were treated with $50,100,200,300$, or $400 \mu \mathrm{g} \mathrm{mL}{ }^{-1} 5$-FU for $48 \mathrm{~h}$. SGC7901/5-FU cells showed resistance to chemotherapy relative to SGC7901 cells (Fig. 2A). To verify the effect of miR-4516 on the drug resistance of gastric cancer cells, we transfected an miR-4516 mimic $\left(50 \mathrm{nmol} \mathrm{L}^{-1}\right)$ into SGC7901 cells and an miR-4516 inhibitor $\left(100 \mathrm{nmol} \mathrm{L}^{-1}\right)$ into SGC7901/5-FU cells. We thus carried out gain-of-function and loss-of-function experiments. Remarkably, upregulation of miR-4516 in SGC7901 cells decreased the apoptosis rate and upregulated the IC50 values (Fig. 2B). In contrast, silencing of miR-4516 increased the apoptosis rate and additionally decreased the IC50 values (Fig. 2B and 1E). Compared with control groups, the effect of the miR-4516 inhibitor on the apoptotic rate was greater in SGC7901/5-FU cells than in control cells $(23.08 \%$ vs. $3.82 \%)$ (Fig. 2B). Downregulation of miR-4516 in SGC7901/5-FU cells sensitized the drug-resistant cells to 5-FU by increasing the level of the apoptosis-related proteins Bax and Caspase-3 (Fig. 2C).

\section{MiR-4516 downregulates ING4 expression by binding to its MRNA $3^{\prime}$-UTR}

When exploring the mechanism by which miR-4516 exerts its biological function, we identified ING4 as a potential target gene for miR-4516 by searching three public databases (miRDB, mirDIP, TargetScan) (Fig. 1C). As shown in Fig. 2C, the miR4516 inhibitor increased ING4 expression. Furthermore, we constructed luciferase reporter vectors containing the $\mathrm{Wt}$ or mutant ING4 3'-UTR sequences (Fig. 3B). When SGC7901/5-FU cells were transfected with the Wt ING4 $3^{\prime}$-UTR, cotransfection with miR-4516 significantly inhibited the luciferase activity. In contrast, the inhibitory action was abrogated in cells co-transfected with the mutant ING4 3'-UTR. These

was upregulated by miR-4516 inhibitor in SGC7901/5-FU cells. ING4 up-regulation partially reversed the miR-4516 mediated inhibition of cell proliferation. (F) SGC7901/5-FU cells were transfected with ING4 plasmid or ING4-NC and incubated with miR-4516 inhibitor $48 \mathrm{~h}$ after transfection. Cell apoptosis was analyzed through flow cytometry to examine the cell apoptosis rate. ${ }^{*} P<0.05$ compared with ING4-NC. 
A

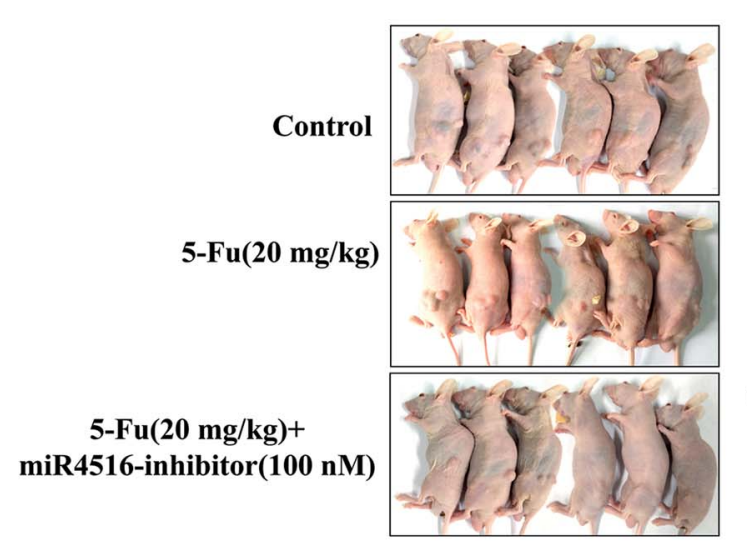

$\mathrm{C}$

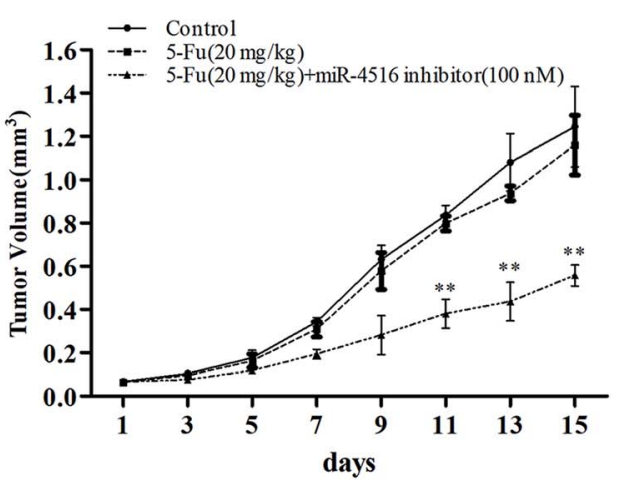

$\mathrm{E}$

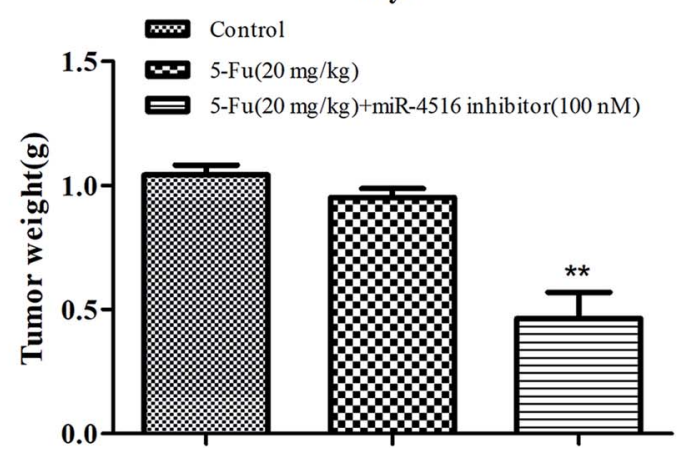

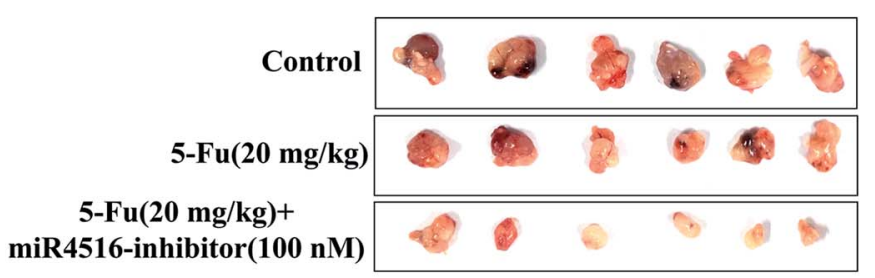

$\mathrm{D}$

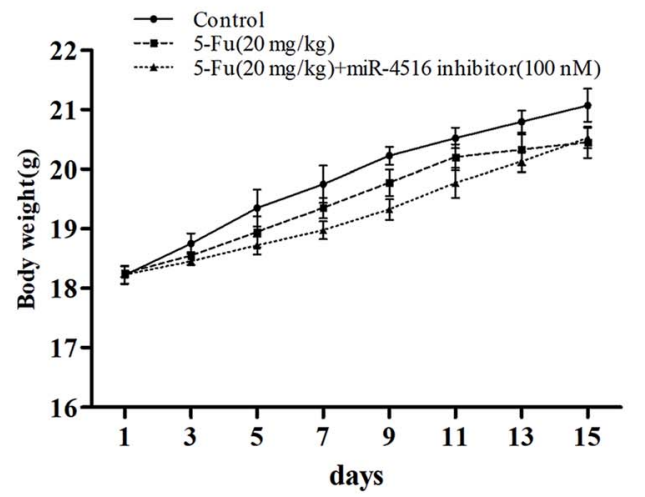

Fig. 5 miR-4516 inhibits tumor growth in GC xenografts in nude mice. SGC7901/5-FU cells stably overexpressing miR-4516 inhibitors combined with 5 -fluorouracil group or negative control were subcutaneously injected into right flank of each nude mouse $(n=6)$. (A and B) Photograph of tumors (C) volumes of all tumors were detected every 3 days $(D)$ body weights of all tumors were detected every 3 days (E) tumor weights of were detected after the dissection. Data are presented as mean \pm S.D; $* * P<0.01$ compared with the Lenti-vector group, Student's $t$-test.

findings suggested that ING4 is a direct target gene of miR-4516 in gastric cancer cells.

\section{Upregulation of ING4 enhances chemosensitivity of gastric cancer cells}

Next, we investigated whether upregulation of ING4 increased sensitivity to chemotherapeutic agents in SGC7901/5-FU cells. To this end, SGC7901/5-FU cells were transfected with an ING4expressing vector or negative control (NC). Western blot confirmed that ING4 expression was markedly upregulated after the transfection of SGC7901/5-FU cells with the ING4-expressing vector (Fig. 3A). We observed decreased IC50 values for 5-FU after transfection of SGC7901/5-FU cells with the ING4-expressing vector (Fig. 3C). Moreover, the function of ING4 in multidrug- resistant gastric cancer cells was verified by the increase in the apoptosis rate under the action of 5-FU in SGC7901/5-FU cells after upregulation of ING4 (Fig. 3D). Taken together, these results suggested that ING4 correlated with MDR of gastric cancer cells, and upregulation of ING4 increased the sensitivity to 5-FU.

Downregulation of ING4 is associated with a poor prognosis and chemoresistance in gastric cancer patients and cell lines

To clarify the clinical significance of ING4 in gastric cancer, we analyzed the data from Kaplan Meier Plotter and Oncomine. We found that ING4 was significantly downregulated in 5-FUresistant groups compared to 5-FU-sensitive groups in independent cohorts (Fig. 4A and B). Furthermore, lower ING4 expression was associated with relatively shorter overall survival 
in a cohort of 578 patients with gastric cancer and shorter progression free survival in a cohort of 426 patients with gastric cancer (Fig. 4C). These findings indicated that ING4 might serve as a biomarker of gastric cancer and that lower expression of ING4 is associated with a poor prognosis. To study the role of ING4 in gastric cancer MDR, the expression of ING4 in samples manifesting different chemosensitivity rates was examined next. To further characterize the function of ING4 in gastric cancer chemoresistance, RT-PCR analyses confirmed that ING4 expression was markedly lower in SGC7901/5-FU cells compared with SGC7901 cells in compendia of cell lines from the Oncomine database (Fig. 4D). Thus, these findings confirmed that low expression of ING4 correlates with gastric cancer MDR.

\section{ING4 is involved in miR-4516-mediated tumor suppression}

Previous research indicates that miR-4516 has a negative effect on gastric cancer chemosensitivity. Because ING4 can inhibit cancer cell chemo-resistance and miR-4516 can regulate the expression of ING4, we hypothesized that miR-4516-mediated upregulation of ING4 would directly reverse gastric cancer chemoresistance. To test this hypothesis, SGC7901/5-FU cells were co-transfected with the miR-4516 inhibitor or miR-Ctrl and either ING4/pcDNA3 plasmid or empty pcDNA3 plasmid as a control. The ING4 expression level was decreased by miR-4516 expression, whereas transfection of the ING4-carrying plasmid restored ING4 expression in SGC7901/5-FU cells in miR-4516 groups. ING4 upregulation reversed the miR-4516-mediated activation of cell proliferation (Fig. $4 \mathrm{E}$ and $\mathrm{F}$ ). These results supported the idea that miR-4516 mediates SGC7901/5-FU tumor progression by inhibiting ING4 expression.

\section{The miR-4516-ING4 axis regulates gastric cancer MDR by regulating apoptosis-associated proteins}

Using western blot assays, we analyzed the expression of antiapoptotic proteins (Caspase-3, Bcl-2) and pro-apoptotic protein Bax in SGC7901/5-FU cells after transfection with the miR-4516 inhibitor or the ING4-expressing plasmid for $48 \mathrm{~h}$ (Fig. 2C and 3A). ING4 is capable of interacting with apoptosisrelated proteins (Bax, Bcl-2, and Caspase-3). Thus, we speculated that ING4 suppresses drug resistance of gastric cancer cells through apoptosis-related proteins. Western blotting was performed to determine whether expression of apoptosisrelated proteins changed after miR-4516 was downregulated. In addition, Bcl-2 and P-gp expression levels were found to be remarkably increased after the ING4 knockdown or miR-4516 upregulation. By contrast, Bax and Caspase- 3 showed the opposite results. In summary, these results indicated that the miR-4516-ING4 axis might regulate gastric cancer cell MDR primarily by increasing apoptosis activity and secondarily by increasing activities of apoptosis-related signaling pathways.

\section{The tumor-promoting activity of miR-4516 in vivo mouse model of a subcutaneous xenograft tumor}

SGC7901/5-FU control group or administration of 5-FU or administration of 5-FU combinated with miR-4516 inhibitortransfected SGC7901/5-FU cells were subcutaneously injected into nude mice, and xenograft tumor growth was monitored. The tumors from administration of 5-FU $\left(20 \mathrm{mg} \mathrm{kg}^{-1}\right)$ or SGC7901/5-FU control group grew significantly faster than administration of 5-FU ( $20 \mathrm{mg} \mathrm{kg}^{-1}$ ) combinated with miR-4516 inhibitor-transfected SGC7901/5-FU cells from 3 days after cell implantation. At 15 days after cell injection, the nude mice were euthanized, and the xenograft tumors were extracted, as depicted in Fig. 5A and B. Next, the tumors were weighed and their volumes were measured. We found that the tumor weight and volume of 5-FU combinated with miR-4516 inhibitor were lower than those in the control groups (Fig. 5C-E). Taken together, these results indicated that miR-4516 plays an oncogenic role in gastric cancer resistance.

\section{Discussion}

Chemotherapeutic drugs, especially 5-FU, are used widely in the treatment of gastric cancer. Nonetheless, this treatment has been hindered by the development of cancer cell resistance to 5-FU. Therefore, the factors affecting sensitivity of gastric cancer cells to chemotherapy have become promising therapeutic targets. Extensive studies have indicated that a miR-4516 inhibitor could become a novel therapeutic tool. In this study, our research focused on the potential usefulness of miR-4516 inhibition for resensitizing gastric cancer cells manifesting drug resistance.

To study the molecular mechanism underlying the resistance of SGC7901/5-FU cells to 5-FU, numerous studies have been carried out. Yan et al. have reported upregulation of nine miRNAs and downregulation of 18 miRNAs in the SGC7901/5FU cell line compared with its parental cells. ${ }^{17}$ In contrast, our experiments yielded different results. Our experimental data revealed that the expression levels of 47 miRNAs in SGC7901/5FU cells were notably higher compared with those of SGC7901 cells. Moreover, 55 miRNAs were found to be downregulated. In 2013, Song et al. identified additional putative targets of miR4516 in multiple pathways firstly, including apoptosis and cell cycle regulation. MiR-4516 and miR-601 appear to correlate and target components of both STAT3 and NF- $\kappa$ B pathways, which are critical regulators of inflammatory responses in cancer. ${ }^{\mathbf{1 4}}$ Several studies suggest that both miR-4516 and miR-601 are involved in the clinical response to rescue radiotherapy after a prostatectomy. ${ }^{15}$ Accordingly, we made a novel finding that miR-4516 expression is increased in gastric cancer cells. After treatment of the cells with the miRNA-4516 inhibitor, the expression of miR-4516 decreased. According to these data, combined with the results of our RT-PCR, we chose miR-4516 for further analysis. We then found that SGC7901/5-FU cells that were transfected with the miR-4516 inhibitor showed reduced resistance to 5-FU. These results suggest that miR-4516 is closely related to the sensitivity of gastric cancer cells to 5-FU.

Moreover, we validated that miR-4516 suppresses ING4 expression. Some reported that the expression of ING4 has been identified as an indicator of docetaxel sensitivity in human lung adenocarcinoma cells. ${ }^{17}$ Another study states that ING4 mRNA is a direct target of miR-423-5p and miR-650. ${ }^{16}$ Nonetheless, there are no reports regarding the relation between ING4 and 5FU resistance. Notably, we confirmed that ING4 plays a critical 
role in the regulation of apoptosis in gastric cancer cells. Strikingly, reintroduction of ING4 enhanced the sensitivity of our gastric cancer cells to 5-FU. By contrast, transfection of these gastric cancer cells with mutant ING4 without a miR-4516binding site did not change 5-FU resistance, suggesting that ING4 plays a critical role in miR-4516-mediated 5-FU sensitivity. Altogether, our findings provide an insight into the molecular basis for 5-FU resistance.

To the best of our knowledge, we are the first group to study the molecular mechanisms of action of miR-4516 in SGC7901/5FU cells with respect to its participation in 5-FU resistance. Overall, the sequential validation experiments suggest that miR4516 may suppress ING4 expression through a direct interaction with the 3'-UTR of its mRNA in gastric cancer SGC7901/5-FU cells. These findings point to a miR-4516-mediated regulatory mechanism of 5-FU chemosensitivity in the SGC7901/5-FU gastric cancer cell line. Our results clearly show that after the downregulation of miR-4516, the sensitivity of SGC7901/5-FU cells to 5FU increased because of the upregulation of the target gene ING4 in these gastric cancer cells. Therefore, the interaction between miR-4516 and ING4 may become a target for reversing 5-FU resistance in gastric cancer. Additionally, miR-4516 may serve as a predictive marker of the response of patients to chemotherapy and as a potential biomarker of gastric cancer.

In summary, our study describes a potential mechanism for dysregulation of ING4 by miR-4516 in gastric cancer. Taken together, downregulation of miR-4516 and activation of ING4 could be a promising approach to improving the treatment of gastric cancer.

\section{Conflicts of interest}

The authors declare that they have no conflicts of interest.

\section{Acknowledgements}

This study was supported by the National Natural Science Foundation of Jiangsu Province (grant no. BK20151016), was supported by the National Natural Science Foundation of China (grant no. 81602441, 81503528), and funded by Training Programme Foundation for the Youth Key Talents by Jiangsu Provincial Commission of Health and Family Planning of China (No. QNRC2016653), and funded by Jiangsu Provincial Key Research Development Program (grant no. BE2016794), and supported by the Talents Program of Jiangsu Cancer Hospital.

\section{References}

1 W. K. Leung, M. S. Wu, Y. Kakugawa, et al., Screening for gastric cancer in Asia: current evidence and practice, Lancet Oncol., 2008, 9(3), 279-287.

2 F. Kamangar, G. M. Dores and W. F. Anderson, Patterns of cancer incidence, mortality, and prevalence across five continents: defining priorities to reduce cancer disparities in different geographic regions of the world, J. Clin. Oncol., 2006, 24(14), 2137-2150.
3 S. A. Hundahl, J. L. Phillips and H. R. Menck, The National Cancer Data Base report on poor survival of US gastric carcinoma patients treated with gastrectomy: fifth edition American Joint Committee on Cancer staging, proximal disease, and the different disease hypothesis, Cancer, 2000, 88(4), 921-932.

4 J. H. Son, T. W. Kong, J. Paek, K. H. Song, S. J. Chang and H. S. Ryu, Clinical characteristics and prognostic inflection points among long-term survivors of advanced epithelial ovarian cancer, Int. J. Gynaecol. Obstet., 2017, 139, 352-357.

5 L. A. Yates, C. J. Norbury and R. J. Gilbert, The long and short of microRNA, Cell, 2013, 153, 516-519.

6 D. P. Bartel, MicroRNAs: target recognition and regulatory functions, Cell, 2009, 136, 215-233.

7 T. Eskildsen, H. Taipaleenmaki, J. Stenvang, B. M. Abdallah, N. Ditzel, A. Y. Nossent, M. Bak, S. Kauppinen, et al., MicroRNA-138 regulates osteogenic differentiation of human stromal (mesenchymal) stem cells in vivo, Proc. Natl. Acad. Sci. U. S. A., 2011, 108, 6139-6144.

$8 \mathrm{~J}$. He, H. Qi, F. Chen and C. Cao, MicroRNA-25 contributes to cisplatin resistance in gastric cancer cells by inhibiting forkhead box O3a, Oncol. Lett., 2017, 14(5), 6097-6102.

9 L. Jiang, W. Yang, W. Bian, H. Yang, X. Wu, Y. Li, W. Feng and $\mathrm{X}$. Liu, microRNA-623 targets cyclin D1 to inhibit cell proliferation and enhance the chemosensitivity of cells to 5-fluorouracil in gastric cancer, Oncol. Res., 2018, 1-25.

10 M. Sui, A. Jiao, H. Zhai, Y. Wang, Y. Wang, D. Sun and P. Li, Upregulation of miR-125b is associated with poor prognosis and trastuzumab resistance in HER2-positive gastric cancer, Exp. Ther. Med., 2017, 14(1), 657-663.

11 X. Y. Zhang, J. Yao, K. Guo, H. Huang, S. yuan Huai, R. Ye, et al., The functional mechanism of miR-125b in gastric cancer and its effect on the chemosensitivity of cisplatin, Oncotarget, 2018, 9(2), 2105-2119.

12 L. Zhou, X. W. Li, F. Zhou, Z. A. Jin, D. Chen, P. Wang, et al., Downregulation of leucine-rich repeats and immunoglobulin like domains 1 by microRNA-20a modulates gastric cancer multidrug resistance, Cancer Sci., 2018, 109, 1044-1054.

13 I. Riquelme, P. Letelier, A. L. Riffo-Campos, P. Brebi and J. C. Roa, Emerging Role of miRNAs in the Drug Resistance of Gastric Cancer, Int. J. Mol. Sci., 2016, 17, 424.

14 S. Song and J. A. Ajani, The role of microRNAs in cancers of the upper gastrointestinal tract, Nat. Rev. Gastroenterol. Hepatol., 2013, 10, 109-118.

15 T. Ishimoto, H. Baba, D. Izumi, et al., Current perspectives toward the identification of key players in gastric cancer microRNA dysregulation, Int. J. Cancer, 2016, 138, 13371349.

16 T. C. Hour, S. D. Chung, W. Y. Kang, et al., EGFR mediates docetaxel resistance in human castration-resistant prostate cancer through the Akt-dependent expression of ABCB1 (MDR1), Arch. Toxicol., 2015, 89, 591-605.

17 Z. Yan, J. Jiang, F. Li, et al., Adenovirus-mediated LRIG1 expression enhances the chemosensitivity of bladder cancer cells to cisplatin, Oncol. Rep., 2015, 33, 1791-1798. 